\title{
Diacronie
}

Studi di Storia Contemporanea

$\mathrm{N}^{\circ} 24,4 \mid 2015$

Le dittature militari: fisionomia ed eredità politica

\section{Dittatura militare e televisione in Brasile: una rilettura critica (1964-1979)}

\section{Sonia Wanderley}

Traduttore: Jacopo Bassi

\section{OpenEdition \\ Journals}

Edizione digitale

URL: http://journals.openedition.org/diacronie/3736

DOI: 10.4000/diacronie.3736

ISSN: 2038-0925

Editore

Association culturelle Diacronie

Notizia bibliografica digitale

Sonia Wanderley, « Dittatura militare e televisione in Brasile: una rilettura critica (1964-1979) »,

Diacronie [Online], $N^{\circ} 24,4$ | 2015, documento 9, Messo online il 29 décembre 2015, consultato il 22 avril 2019. URL : http://journals.openedition.org/diacronie/3736 ; DOI : 10.4000/diacronie.3736 


\title{
Diacronie
}

N. 24 | 4|2015 Le dittature militari: fisionomia ed eredità politica

\section{9/}

\section{Dittatura militare e televisione in Brasile: una rilettura critica (1964-1979)}

\author{
Sonia WANDERLEY *
}

traduzione a cura di Jacopo BASSI

Nonostante l'autoritarismo, la dittatura militare brasiliana produsse un discorso volto alla difesa della democrazia, conformemente alla Dottrina della Sicurezza Nazionale (DSN). Per legittimarla, la programmazione televisiva assunse un ruolo pedagogico nel consolidamento dei concetti cari al regime, principalmente quelli di integrazione nazionale e di modernità. Questo saggio mostra come il settore televisivo fosse parte integrante del gruppo che assunse il potere politico nel paese nel 1964. Tuttavia, come altre forme di solidarietà, quella stabilitasi tra i proprietari delle emittenti televisive (una concessione pubblica) e la dittatura militare brasiliana non fu esente da rotture. L'articolo evidenzia la necessità di riconsiderare le letture esistenti che hanno voluto vedere, semplicisticamente, un'alleanza fra questi ambienti e la dittatura militare.

\section{Presentazione: $\mathrm{i}$ termini della questione}

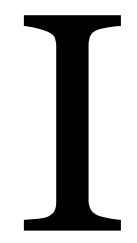

n Brasile il processo di formazione di una cultura di massa è normalmente datato alla fine degli anni Venti, inizio degli anni Trenta. Fu il momento in cui le trasformazioni di ordine economico e politico del paese, assieme alla crescita dei centri urbani e alla crescente immigrazione permisero l'organizzazione di una società che «inizia ad essere di "massa" ed entra in collisione con la società "normalizzata" nella sua segregazione di classe e tra i gruppi sociali».

${ }^{1}$ MARTIN-BARBERO, Jesús, Dos meios às mediações: comunicação, cultura e hegemonia, Rio de Janeiro, Editora da UFRJ, 2009, p. 216. 
In questo contesto si ridefinirono le relazioni tra Stato e società civile, dando origine a un progetto nazionale che aveva l'obiettivo di far avanzare il paese verso la modernità per mezzo dell'industrializzazione e, necessariamente, a partire dall'incorporazione di segmenti delle classi popolari nello scenario politico. Dal momento che un tale progetto non si costruì sotto il segno della rottura totale con l'antico ordine politico egemonico, ma da movimenti di adattamento, il processo di inclusione di queste classi si svolse, in ultima istanza, in modo controllato.

Lo Stato e la cultura emergono tra gli elementi che concorrono al conseguimento del progetto nazionale in essere. Al primo tocca il ruolo di omogeneizzatore delle differenze e di arbitro dei conflitti sociali. La seconda, svolge la funzione di rilevante materia prima nella costruzione dell'unità idealizzata intorno al significato di nazione e nazionalità.

Così, per la prima volta nella storia del paese, la questione culturale, sebbene passando necessariamente attraverso valori popolari, iniziò a permeare la politica. La dinamica della politica culturale in essere, principalmente per il ruolo che compete allo Stato, si plasmava su quella dell'economia politica. Questo spiega l'importanza attribuita dalle politiche pubbliche a quella che veniva chiamata industria culturale giornali cinema e, soprattutto, radio - già negli anni Trenta e Quaranta.

La televisione venne introdotta in Brasile all'inizio degli anni Cinquanta e, circa un decennio dopo, già era in grado di prendere il posto che fino ad allora aveva avuto la radio, trasformandosi nella più importante espressione della cultura popolare di massa. Ciò significa che divenne la principale rappresentante di un'ibridazione tra il campo della cultura artistica, della produzione limitata, e della sfera culturale del mercato, della produzione su ampia scala ${ }^{2}$. Questa posizione trasferì in essa un capitale simbolico, facendone un agente fondamentale nella produzione di significati sociali e politici.

L'inizio delle trasmissioni televisive nel paese venne visto come un segnale dell'ingresso nella modernità. Tuttavia a quell'epoca questa espressione aveva già cessato di avere un significato legato solamente all'idea di nazione, come era stata tratteggiata dagli intellettuali degli anni Trenta e Quaranta. La congiuntura del Secondo dopoguerra produrrà infatti una discussione sulle condizioni necessarie per lo sviluppo di tutti i popoli. In questo dibattito, il miglioramento delle comunicazioni era

\footnotetext{
2 Per un approfondimento del concetto "cultura populare di massa", si veda: ORTIZ, Renato, $A$ moderna tradição brasileira - cultura brasileira e indústria cultural, São Paulo, Brasiliense, 1995; CANCLINI, Nestor Garcia, Culturas híbridas - estrategias para entrar y salir de la modernidad, México, Grijalbo, 1990.
} 
considerato come prioritario per l'integrazione della nazione nel processo di sviluppo. In Brasile gli studi sull'identità nazionale subivano l'influenza di questo dibattito.

A partire dagli anni Cinquanta, assieme con la crescita della febbre dello sviluppo, si intravedeva un altro progetto di nazione per il Brasile. Secondo gli intellettuali militanti sostenitori di questa visione politica, il cammino per la modernità e il conseguente sviluppo, sarebbe passato necessariamente dalla crescita industriale, dal momento che l'aumento della produzione di beni manufatti avrebbe condotto, pragmaticamente, alla crescita del consumo e a una redistribuzione della ricchezza, preparando la strada per la democrazia. In questo sistema, il ruolo del capitale straniero ha un peso decisivo e, mentre disegna la forma con cui il paese si inserisce nell'economia capitalista internazionale, conferisce nuove sfumature ai discorsi sull'identità nazionale.

L'euforia nei confronti dello sviluppo rimase anche quando le strategie tipiche del nazional-populismo ${ }^{3}$ iniziarono a non tener più conto delle rivendicazioni sociali che sorgevano e la coesistenza proposta dalla Stato con queste ultime si sfilacciò. Per garantire la continuità del modello di sviluppo impiantato nel paese, le élites dominanti misero mano ai meccanismi istituzionali della democrazia-liberale e, nel 1964, appoggiarono un golpe militare. In quel momento i mezzi di comunicazione di massa vennero deviati dalla loro funzione politica e divennero appannaggio dell'apparato economico4. La televisione, tuttavia, come espressione più compiuta della cultura popolare di massa, giocherà un ruolo fondamentale nel processo di integrazione, sviluppo e sicurezza, istituito dal regime politico che si insatura nel paese.

Questo protagonismo della televisione può essere spiegato a partire da due aspetti. In primis, è frutto delle profonde trasformazioni nell'organizzazione industriale brasiliana che si verificarono a partire dal 1964. La crescita delle infrastrutture industriali permise il consolidamento di un mercato dei beni culturali. Il milagre econômico portò, tra le altre cose, l'interazione tra lo Stato e lo sviluppo dell'industria delle telecomunicazioni, ponendo le basi necessarie per lo sviluppo della televisione su di una rete nazionale, così come per l'avvento della tecnologia della TV a colori. Da un altro punto di vista, i migliori livelli salariali e la facilità di credito per le classi medie consentirono un'espansione delle vendite degli apparecchi televisivi.

\footnotetext{
3 Senza addentrarci in ulteriori polemiche riguardo alla corrente applicazione del concetto di populismo ai regimi instauratisi nel paese entrar em maiores polêmicas acerca da coerência da aplicação do conceito populismo aos regimes instalados no país dopo la caduta dell'Estado Novo (1937-45), ci preme qui puntualizzare come in questo modello la presenza fondamentale dello Stato assicurando la modernità e lo sviluppo, garanti dell'unità nazionale, assumendosi i compiti di istituire e, allo stesso tempo, controllare gli attori collettivi, mediare i conflitti e produrre una democrazia "reale", in opposizione a una democrazia "formale", vista generalmente come un sistema destinato a favorire la perpetuazione dei privilegi.

${ }_{4}$ Cf. MARTIN-BARBERO, Jesús, op. cit.
} 
In secondo luogo, la televisione rifletté in qualche modo un affinamento qualitativo dei dispositivi ideologici. Questa, tra tutti i veicoli della cultura di massa esistenti, fu quella che seppe produrre un'unificazione della domanda di beni, tanto quelli materiali quanto quelli simbolici, rispondendo al progetto del regime militare, prodotto di una dottrina che metteva in relazione sviluppo e sicurezza: la Dottrina della Sicurezza Nazionale (DSN).

Uno dei due assi fondamentali del progetto del regime militare consisteva nell'unificazione culturale. Sottesa al discorso dell'unità culturale - che nascondeva le differenze socioeconomiche del paese - si trovava una proposta di modernità che trovava nel consumo il suo sostentamento. Oltre a divertire, informare e istruire, la televisione brasiliana favoriva la concretizzazione degli obiettivi del capitalismo dipendente che era instaurato in Brasile sia fornendo nuove alternative allimpiego del capitale, sia agendo come veicolo per la valorizzazione dei beni di consumo prodotti attraverso la trasmissione della pubblicità5. Nello stesso tempo in cui facilitava l'integrazione economica, l'unificazione culturale rendeva possibile la formazione di un consenso allargato riguardo ai valori e agli ideali che avrebbero dato forma alla "reale" identità nazionale.

L’integrazione nazionale, concetto chiave della Dottrina della Sicurezza Nazionale, finiva dunque per confondersi con l'unificazione culturale e con la creazione di un solo pubblico - con l'intento di far svanire le differenze regionali - fossero esse materiali o culturali, facilitando la creazione di un mercato nazionale di beni materiali così come di beni culturali e simbolici. Siamo sviluppati - questa era la considerazione - se siamo in grado di consumare nella stessa misura dei paesi centrali del sistema capitalista, concretamente e simbolicamente.

\section{Cultura nazionale e integrazione}

$\mathrm{Fu}$ in un momento di intenso fermento culturale che si verificò il golpe civile militare brasiliano del 1964. Il cinema, il teatro, la letteratura e la musica popolare brasiliana vivevano un periodo di effervescenza, caratterizzato dalla creatività, dall'audacia e, soprattutto, per la fiducia nella capacità popolare di cambiare non solamente l'arte, ma l'intera società. L'egemonia della retorica di sinistra dava vita all'arte impegnata, intenta nel compito di rendere cosciente il popolo brasiliano, oppresso e derubato, conducendolo verso la rivoluzione.

5 CAPARELLI, Sérgio, Televisão e Capitalismo no Brasil, Porto Alegre, LP\&M, 1982. 
In qualche modo tutta questa mobilitazione rifletteva una profonda incompatibilità tra il modello politico-economico instaurato nel paese e le forze sociali che questo stesso modello continuava a rafforzare. Il patto nazional-popolare aveva i giorni contati. La crisi economica iniziata nel 1962 aveva reso più evidente la differenza fra le classi sociali, che pure lo Stato sino ad allora aveva cercato di livellare. Per altri versi impediva che alle crescenti domande popolari venisse data risposta, un fondamento dello Stato che si era consolidato alla fine della dittatura dell'Estado Novo. Ma dal momento che erano divenute più evidenti le contraddizioni del patto, risultò più difficile il mantenimento dell'ordine economico, politico e sociale necessario al consolidamento del sistema capitalista nel paese ${ }^{6}$. Il golpe che si verificò nel 1964, dunque, riunì i settori civili e militari in cerca di una ridefinizione del patto di potere.

Il contesto interno di svuotamento del patto politico fino ad allora sussistente era aggravato dalla bipolarizzazione ideologica degli anni Sessanta, che incoraggiava le letture autoritarie, ricorrenti nell'immaginario politico nazionale, in relazione alle forme e alle pratiche di organizzazione e di rivendicazione dei lavoratori. Infatti, il rafforzamento dei sindacati, gli scioperi per il salario, gli scontri con la polizia, le proteste pubbliche in generale e quelle studentesche in particolare, erano identificate come disordine e caos. Il disordine sociale era visto come effetto dell'incapacità del governo e dell'influenza nefasta dei comunisti, infiltrati negli organi pubblici e di classe: questo impediva l'“evoluzione" della nazione, nella misura in cui allontanava il capitale straniero, fondamentale per il consolidamento del modello di sviluppo capitalista difeso dal nuovo blocco di potere che andava costituendosi.

In questa congiuntura la struttura dottrinale che sostenne la reinterpretazione del processo di consolidamento in Brasile nacque dall'accordo fra tre enti che formarono il blocco delle forze golpiste: la Escola Superior de Guerra (ESG), l'Instituto de Pesquisas e Estudos Sociais (IPÊS) e l'Instituto Brasileiro de Ação Democrática (IBAD)7.

In questo triangolo, toccarono rispettivamente all'IPÊS e all'IBAD, le funzioni di diffusione delle idee $^{8}$ e di distribuzione dei fondi - principalmente provenienti dalle

\footnotetext{
${ }^{6}$ MENDONÇA, Sonia Regina, Estado e economia no Brasil: opções de desenvolviment, Rio de Janeiro, Graal, 1986, p. 69.

7 Cfr. DREIFUSS, René Armand, 1964: a conquista do Estado - ação política, poder e golpe de classe, Petrópolis-Rio de Janeiro, Vozes, 1981.

${ }^{8}$ Come organo essenziale della propaganda, l'IPÊS giocò un ruolo fondamentale nella diffusione di documenti, nell'organizzazione di seminari e nella produzione di film tra gli imprenditori, nei sindacati, nelle associazioni studentesche ed anche fra le casalinghe, irregimentate e organizzate dalla Campanha da Mulher pela Democracia (CAMDE), il suo braccio femminile. Spettò a questo anche il compito di organizzare un "Servizio di Ricerca e Informazioni", producendo un archivio che servì da embrione per la fondazione del Serviço Nacional de Informações (SNI), creato una volta realizzato il golpe militare.
} 
multinazionali -, per azioni che miravano a legittimare il tentativo di golpe contro il governo costituzionale del presidente João Goulart9. L'ente principale, dove tutto veniva plasmato era, sostanzialmente, la ESG.

Sin dalla fondazione, nel 1949, la ESG lasciò trasparire una finalità pedagogica, quella di trasformarsi in un centro di studi dei problemi brasiliani, riunendo intellettuali ed altri professionisti - civili così come militari -, con l'obiettivo formulare un metodo di pianificazione per le azioni dello Stato e dotare le élites del paese di una maggior capacità di amministrazione, come risulta dai manuali prodotti dal suo Dipartimento di Studio:

I grandi movimenti della storia brasiliana furono sempre manifestazioni delle élites. La campagna abolizionista, l'Indipendenza, la Repubblica ne sono chiari esempi. L'adesione del popolo alle azioni d'avanguardia e di sostegno a questi movimenti sono sitate così modeste che non è riscontrabile una partecipazione degna di nota [...]. Alcuni fattori come le difficoltà di comunicazione e l'estensione territoriale hanno concorso a ciò. Detto questo, la congiuntura ha fatto sì che questi eventi passassero attraverso decisioni dei vertici. [...] $\mathrm{Ci}$ sembra che laStoria Brasiliana dia rilievo al ruolo delle Élites nella enunciazione degli Obiettivi Nazionali $[\ldots]^{10}$.

Anche quando la «partecipazione politica del popolo alla vita nazionale è più sensibile», afferma lo stesso manuale, non deve essere diminuita la responsabilità delle élites del paese. Tuttavia queste devono essere identificate «concretamente con gli Obiettivi Nazionali» ${ }^{11}$. I loro compiti continuano a essere quelli di identificare e interpretare correttamente gli interessi e le aspirazioni del popolo proteggendo quelle che rappresentano in maniera veritiera le radici storico-culturali brasiliane, così come quello di condurre ad un perfezionamento materiale e spirituale della nazione per mezzo della promozione di nuovi obiettivi e aspirazioni coincidenti con il percorso di sviluppo nazionale.

Per la ESG, tuttavia, le élites militari avevano funzioni differenti da quelle civili. A queste ultime toccava interpretare le aspettative della società e farle realizzare allo

\footnotetext{
${ }^{9}$ La relazione tra le due istituzioni divenne palese al momento della chiusura dell'IBAD, dopo essere stato investigato dalla stessa Commissione parlamentare di inchiesta che indagò sulle attività dell'IPÊS nel 1963. Le irregolarità riscontrate evidenziavano come questo ricevesse fondi dall'estero senza un'autorizzazione ufficiale.

${ }^{10}$ Manual Básico - Política Nacional, Rio de Janeiro, Departamento de Estudos/ESG, 1975, pp. 49-50.

${ }^{11}$ Ibidem, p. 50.
} 
Stato. Avevano anche il dovere di identificare i valori e i comportamenti del popolo che dovevano essere rafforzati o eliminati. Ma, ritenevano gli intellettuali della Scuola, la storia del paese comprovava come l'azione delle élites civili fosse stata marcata dall'empirismo, dall'improvvisazione e dall'individualismo. Per questo motivo, spettava ai militari la responsabilità pedagogica di dotarle di strumenti che realizzassero il compito di interpretare correttamente - per una miglior conoscenza della realtà socioculturale e dei dati congiunturali - le ansie e le aspirazioni dei differenti gruppi sociali che componevano la nazione, armonizzandoli in modo che rispondessero al bene comune.

Coloro che erano parte integrante della Scuola discutevano la necessità dell'introduzione di un processo di razionalizzazione politica, di pianificazione, capace di creare le condizioni necessarie perché le élites potessero

[...] affrontare le sfide che attendevano lo Stato brasiliano, soprattutto quelle legate, da un lato all'eliminazione della minaccia comunista e, dall'altro, alla promozione dello sviluppo del paese. Nell'affrontare queste sfide sarà necessario non solamente formulare [...] "un nuovo progetto per la società e per lo Stato brasiliano", ma anche dotare le élites e la popolazione brasiliana di "un'altra visione del mondo"12.

Pertanto la funzione dell'élite militare, formata secondo questi precetti, non sarebbe stata solamente quella di costruire gli strumenti metodologici per la pianificazione dell'azione della Stato. Avrebbe avuto, oltre a questo compito, l'obbligo di fornire idee, tenendo in considerazione l'istituzione di nuovi modelli di comportamento, tanto per l'élite civile così come per le classi lavoratrici. Per questo motivo sarebbe stata necessario l'innalzamento delle «condizioni di sviluppo della nazione e le sue risorse culturali» ${ }^{13}$.

La proposta articolata dalla ESG non si limitava, come vedremo, all'elaborazione di un manuale tecnico. Il carattere dottrinario di questi studi traspare dalla preoccupazione nel rimarcare la necessità di elaborare strumenti basati su contenuti filosofici e scientifici per l'analisi delle congiunture e fondati tanto sulla conoscenza

${ }^{12}$ Affermazioni del generale Cordeiro de Faria, primo comandante della ESG. Apud ROCHA, Maria Selma de Moraes, A ditadura militar no Brasil: repressão e pretensão de legitimidade 1964-1984, Tesi di Dottorato in Sociologia - Universidade de São Paulo, São Paulo, 1996, pp. 3032.

${ }_{13}$ GURGEL, José Alfredo Amaral, Segurança e democracia: uma reflexão política sobre a doutrina da Escola Superior de Guerra, Rio de Janeiro, Biblioteca do Exército \& Livraria José Olympio Editora, 1975. p. 33. 
della realtà del paese, quanto sui valori etici e morali che erano legati all'origine sociostorica brasiliana.

Per Maria Selma Rocha, gli studi sulla DSN mettono in chiaro la condizione che determinati temi assumono in quanto «idee-forza, intorno alle quali si strutturano i concetti e la Dottrina stessa» ${ }^{14}$. Tra queste tematiche, ci interessa discutere la definizione stabilita per la società e come, a partire da questa, la ESG forgiò la sua teoria politica.

Per gli ideologi della ESG, «la vita sociale è un mandato imperioso della natura umana». Risultato naturale dell'evoluzione dell'uomo, veniva considerata come «ogni gruppo umano che convive sotto una certa struttura sociale, determinata da interessi comuni, in accordo con i valori fissati da un processo storico-culturale» ${ }^{15}$.

Inoltre, evolvendo e facendolo in modo del tutto naturale, la sociabilità dei gruppi in questa fase di sviluppo avrebbe fatto sorgere e crescere un sentimento di solidarietà che avrebbe portato a un «perfezionamento della vita della comunità» dando origine alla nazione:

[...] La società - già sedimentata dall'aver coltivato lungamente tradizioni, costumi, lingua, idee, tendenze -, legata a un determinato spazio di terra e unita dalla solidarietà generata dalle lotte e vicissitudini comuni, che si traduce nella volontà di continuare a vivere assieme e di proiettarsi nel futuro preservando i valori consolidati e cercando la realizzazione degli obiettivi corrispondenti ${ }^{16}$.

Il passo successivo di questa linea di sviluppo sarebbe lo Stato, entità di natura politica, concepito a partire dalla «presa di coscienza della necessità di ricerca di unità». L'«affermazione di un potere sovrano» e «una precisa delimitazione territoriale ${ }^{17}$ sono i suoi elementi fondamentali. Come «conduttore della nazione» ${ }^{18}$, lo Stato abbisogna di stabilire i mezzi che possano permettergli di realizzare le aspirazioni nazionali. Da qui la necessità dell'elaborazione, che fa la Dottrina, di strumenti concettuali, che possano essere utilizzati dallo Stato - considerando la necessaria pianificazione dell'azione - per comprendere la realtà e le necessità del paese, ovvero le aspirazioni nazionali.

\footnotetext{
14 ROCHA, Maria Selma de Moraes, op. cit., p. 56.

15 Escola Superior de Guerra, Complementos da Doutrina, Rio de Janeiro, ESG, 1981, p. 19.

${ }^{16}$ Ibidem, pp. 20-21.

17 Ibidem, p. 21.

18 ESCOLA SUPERIOR DE GUERRA, Fundamentos da Doutrina, Rio de Janeiro, ESG, 1981, p. 20.
} 
Le aspirazioni nazionali sarebbero i bisogni e le necessità, proprie di tutta la società, che si «inseriscono e si proiettano nella coscienza nazionale, nella classe dirigenti e in quella diretta»19. Tali aspirazioni si trasformano in "Obiettivi Nazionali" quando sono mediate dalla azione delle élites dirigenti per mezzo di un metodo di pianificazione.

Definiti i presupposti filosofici, la Dottrina passa a presentare gli strumenti per mezzo dei quali possono essere apprese le reali aspirazioni nazionali, ossia, i concetti che serviranno come base per l'elaborazione e l'esecuzione di un metodo di pianificazione che dovrà essere utilizzato dalle élites per trasformare queste aspirazioni in "Obiettivi Nazionali". Sono questi strumenti concettuali che permettono di capire come, in alcuni casi, taluni obiettivi siano passibili di modifiche (Obiettivi Nazionali mutevoli), seppur mantenendo saldi i valori nazionali strutturali (Obiettivi Nazionali permanenti).

Da questa sintesi si comprende come la Dottrina della ESG abbia costruito un discorso, ritenuto scientifico, per mezzo del quale le trasformazioni storico-sociali furono viste come parte di un'evoluzione naturale, responsabile della situazione contingente. Questa interpretazione organicista considera innaturale tutto ciò che possa disturbare l'“ordine naturale” dello sviluppo di una società o comprometta la sua sopravvivenza e che deve, pertanto, essere eliminato in nome del "bene-comune". Provvedere a questa eliminazione sarebbe uno dei compiti, tra gli altri, delle élites nazionali.

La concezione teorica elaborata non contempla l'idea di rottura. Il carattere strumentale dell'utilizzazione della storia, si rivela attraverso la valorizzazione del concetto di sicurezza nazionale senza una riflessione sul carattere contradditorio, marcato dal conflitto, del processo di costituzione degli interessi sociali.

Fu infatti a partire dal 1953, un momento marcato dall'inasprimento della Guerra fredda e dall'adesione del paese al cosiddetto blocco occidentale, sotto la leadership degli Stati Uniti, che il tema "sicurezza" iniziò ad essere valorizzato ${ }^{20}$. Nella seconda fase dell'esistenza della Scuola, che giunge fino all'anno $1967^{21}$, venne ampliata quella che sarebbe divenuta la sua missione fondamentale: la pianificazione della sicurezza nazionale. Prendendo in considerazione il fatto che la miseria di una parte significativa della popolazione brasiliana si trasformava in terreno fertile per la disseminazione di

19 GURGEL, José Alfredo Amaral, op. cit., pp. 69-77.

${ }^{20}$ L’inizio di questo processo è segnato dalla conferenza "A Segurança Nacional, a Política e a Estratégia: Conceituações e Inter-relações”, tenuta dal generale Juarez Távora, comandante della ESG, nel 1953.

${ }^{21}$ Cf. ARRUDA, Antônio de, A Escola Superior de Guerra: história de sua doutrina, São Paulo, Editora GRD/INL, 1983, pp. XXXV-XXXVI. 
ideali comunisti, cominciò a considerare il binomio sicurezza e sviluppo come centrale nei suoi studi, dando vita alla Dottrina di Politica Nazionale, che copriva i campi della sicurezza e dello sviluppo. La difesa del reale "carattere nazionale" risiedeva nella sostegno offerto ai cambiamenti.

Pur comparendo nei testi della dottrina sin dal 1953, il concetto di "carattere nazionale" divenne autonomo solamente nel 1963, come uno dei «fondamenti psicosociali del Potere Nazionale». In questo frangente il concetto fu così definito:

[...] la stratificazione delle tendenze individuali che determinano la sua condotta sociale. [...] la media dei singoli caratteri costituisce il Carattere Nazionale, che identifica i tratti dominanti di ciascun popolo. La natura di questo elemento è più psicosociale che biologica, poiché fa riferimento al comportamento dell'individuo proiettato nella società 22 .

A partire dal 1964, anno del golpe civile-militare, il concetto iniziò a essere considerato una delle condizioni di partenza per la formulazione degli Obiettivi Nazionali Permanenti. Dai dibattiti sorti a partire da quel momento, la sua definizione acquisisce contorni culturali nel 1971:

[...] Il Carattere Nazionale non è [...] una semplice generalizzazione delle caratteristiche. Nella sua formulazione, si ha un processo storico-culturale, in cui anche le origini della popolazione, le etnie, l'ereditarietà, l'apprendimento, l'educazione e altri elementi concorrono a plasmare la sua struttura $\mathbf{2 3}$.

La Dottrina della ESG, sin dal 1967, definisce così il carattere nazionale brasiliano ${ }^{24}$ :

${ }^{22}$ Ibidem, p. 174.

23 Ibidem, p. 177.

24 Dopo il 1967, vi furono piccoli cambiamenti per ciò che riguarda le qualità di base che non dimostrano comunque un mutamento nella metodologia della definizione. La ESG iniziò a considerare, con finalità didattiche, le seguenti caratteristiche come tipiche del Carattere Nazionale Brasiliano: individualismo, adattabilità, improvisazione, vocazione pacifista, cordialità ed emotività. Cfr. ARRUDA, Antônio de, op. cit., p. 196. 


\begin{tabular}{|c|c|}
\hline \multicolumn{2}{|c|}{ Carattere Nazionale Brasiliano } \\
\hline $\begin{array}{l}\text { Qualità di } \\
\text { base }\end{array}$ & Qualità complementari \\
\hline Individualismo & $\begin{array}{l}\text { Instabilità emozionale (apporto } \\
\text { portoghese) }\end{array}$ \\
\hline Sentimentalismo & $\begin{array}{l}\text { Tendenza alla generalizzazione } \\
\text { (apporto portoghese) }\end{array}$ \\
\hline Adattabilità & $\begin{array}{l}\text { Rispetto per le credenze religiose e le } \\
\text { fedi (apporto portoghese) }\end{array}$ \\
\hline Improvvisazione & $\begin{array}{l}\text { Sentimento di amore per la libertà } \\
\text { (apporto indígena) }\end{array}$ \\
\hline Cordialità & $\begin{array}{l}\text { Astrazione, senso estetico e poetico } \\
\text { (apporto africano) }\end{array}$ \\
\hline Comunicabilità & $\begin{array}{l}\text { Idealismo e tendenza alla teorizzazione } \\
\text { (apporto tedesco) }\end{array}$ \\
\hline \multirow{3}{*}{$\begin{array}{l}\text { Vocazione } \\
\text { pacifista }\end{array}$} & $\begin{array}{l}\text { Esuberanza nell'espressione, culto delle } \\
\text { arti (apporto italiano) }\end{array}$ \\
\hline & Senso critico e umorismo irriverente \\
\hline & Cultura dell'ornamento \\
\hline
\end{tabular}

L'analisi di queste qualità è un dato indicativo importante di una visione del mondo che si ripresenta in diversi momenti della storia brasiliana. A livello di idee questa definizione si basa su uno specifico inconscio collettivo permeato di archetipi mitologici. In prospettiva antropologica, il mito è una narrazione che funziona come soluzione immaginaria per i conflitti che non ne trovano una nel campo della realtà. Per mezzo del mito si costruisce un'unità immaginaria che facilita la fissazione di un ordine per conseguire determinati obiettivi. In questo percorso la Dottrina della ESG riscatta diversi elementi di una «tradizione ottimista» ${ }^{25}$ già presente tra gli intellettuali e nella letteratura del paese, ambendo a legittimare il suo progetto di Sicurezza e Sviluppo.

In questo progetto, quello brasiliano è, in una certa misura, differente e simile ad altri popoli: indipendente e flessibile, razionale e impulsivo. Riassemblando in modo peculiare gli elementi che già componevano l'immaginario collettivo, la Dottrina ingenera una nuova personalità e questo processo potrebbe ripetersi all'infinito, poiché sembra sempre fondare qualcosa di nuovo.

25 Secondo l'analisi di FICO, Carlos, Reinventando o otimismo - ditadura, propaganda e imaginário social no Brasil, Rio de Janeiro, Fundação Getúlio Vargas, 1997. 
L'obiettivo di qualsiasi dottrina che si esprime attraverso un discorso ${ }^{26}$ è di trasformarsi in prassi grazie all'impiego di mezzi adeguati per la sua diffusione. È così che può costituire un fenomeno di massa: fu questa l'intenzione degli ideologi della ESG. Anche se non conseguirono i loro obiettivi, approfondirono il concetto di azione pedagogica dello Stato, servendosi anche del patrocinio della cultura, per diffondere le loro idee tra i settori dell'élite e della classe media urbana, cosa che può essere interpretata come l'intenzione di stabilire l'egemonia per il loro progetto politico.

Nell'affermazione di una dimensione pedagogica nell'intervento dello Stato nella società brasiliana, i governi militari svilupparono - in forma sufficientemente efficace politiche culturali che pur acquistando differenti sfumature, mantenevano, però, affinità quanto alla fiducia nella capacità dei processi culturali di suscitare adesione e di procacciare consenso. L’importanza di procedere in questo modo era già stata esplicitata dalla stessa ESG, già negli anni Cinquanta, da un personaggio che sarebbe divenuto uno dei principali nomi della politica economica del regime militare, Roberto Campos:

[...] Assume particolare importanza nei paesi sottosviluppati la creazione di una "mistica dello sviluppo" capace di provocare forme di comportamento sociale che conducano all'accettazione dei sacrifici connessi a qualsiasi sforzo nell'accelerazione della formazione di capitale nelle economie poco sopra il livello della semplice sussistenza ${ }^{27}$.

In altre parole le politiche culturali portate avanti dal regime militare furono strumenti importanti per l'affermazione di un insieme di rappresentazioni, soprattutto quelle che plasmavano il concetto di “carattere nazionale brasiliano”. Queste aiutavano il regime, nei contesti più disparati, nel compito di organizzare la società brasiliana, secondo quell'insieme di comportamenti, norme, credenze che caratterizzarono fino a quel momento i gruppi che avevano raggiunto il potere politico nel 1964. Lo studio di queste politiche culturali, delle loro caratteristiche e delle modalità con cui vennero attuate nella società ci permette di conoscere qualcosa di più della cultura politica condivisa dall'élite politica dell'epoca.

${ }^{26}$ FOUCAULT, Michel, A ordem do discurso, São Paulo, Edições Loyola, 1996, p. 8.

${ }_{27}$ Cit. in GURGEL, José Alfredo Amaral, Segurança e democracia: uma reflexão política sobre a doutrina da Escola Superior de Guerra, Rio de Janeiro, Biblioteca do Exército \& Livraria José Olympio Editora, 1975, p. 37. 


\section{La televisione sulla scena culturale}

Negli anni Sessanta, benché non fosse possibile negare il contributo della radio e della televisione nel processo culturale brasiliano, la maggior parte degli intellettuali del paese continuava a considerare i mezzi di comunicazione propri della cultura di massa semplicemente come strumenti di manipolazione e di alienazione culturale.

Il riduzionismo di questa posizione, comune fino agli anni Settanta, è stato definito frutto di «eccessiva ideologizzazione e politicizzazione della cultura». Per l'autrice, il discorso accademico e istituzionale sulla cultura superò le politiche culturali autoritarie prodotte da una visione di classe, solamente quando introduce nello stesso discorso la dimensione della fantasia, del ludico e della gratuità 28 .

Il grande equivoco in cui caddero questi intellettuali fu quello di non aver compreso che all'epoca si era già consolidato, in Brasile, un mercato dei beni culturali. La mancanza di questa coscienza gli fece sottovalutare la forza della costruzione simbolica dei mezzi di comunicazione di massa. Se il dibattito sulla "cultura popolare" e sulla "cultura brasiliana" si rivelò essere un tratto costitutivo del percorso intellettuale collettivo del paese, vi fu un silenzio - che sia stato concettuale o che sia stato speculativo - sulla "cultura popolare di massa" che durò sino agli anni Settanta29.

Il disprezzo che traspare in queste analisi deve essere considerato come una lettura accademica che impiegava chiavi di interpretazione incapaci di dare conto di un oggetto come i prodotti culturali della società di massa. Questi erano il risultato di un tipo di organizzazione sociale che entrava in "conflitto con la società "normalizzata" nella sua segregazione tra classi e gruppi sociali», modello esemplare delle letture proposte. Certamente le caratteristiche della lotta politico-ideologica condotta sino a quel momento impedivano di constatare come stessa costituendosi una nuova dinamica per la politica culturale brasiliana che, fondendosi con l'economia politica, puntava alla creazione di un nuovo tipo di relazioni nel processo politico-culturale ${ }^{30}$. I mutamenti sociali, sorti dalla metà degli anni Cinquanta, delineavano una presenza non omogenea della lotta di classe nel paese. Il nuovo modo di essere del "popolare", come afferma Barbero, esigeva un altro tipo di interpretazione, mai apparso sino ad allora tra le letture intellettuali sul popolare e la sua cultura.

${ }^{28}$ FADUL, Anamaria, Políticas culturais e processo político brasileiro, in MELO, José Marques de (org.), Comunicação e transição democrática, Porto Alegre, Mercado Aberto, 1985, pp. 180209, p. 208.

29 ORTIZ, Renato, A moderna tradição brasileira - cultura brasileira e indústria cultural. São Paulo, Brasiliense, 1995 .

30 BARBERO, Jesús Martin, Dos meios às mediações. Comunicação, cultura e hegemonia. Rio de Janeiro, Editora UFRJ, 1997, p. 216. 
La massificazione delle società colpì tutte le classi, ma in modo differente. Le "classi alte", benché continuassero a considerare le rivendicazioni di massa come pericolose, appresero rapidamente a riconoscere il potenziale di "stimolo economico" derivante dalla loro crescita. D'altro canto disprezzavano, ritenendoli privi di stile, i beni materiali e culturali derivati da questo processo di massificazione. Le "classi medie, piccolo-borghesi”, benché condividessero questo disprezzo non erano in condizione di poter prendere le distanze da questa produzione. Per loro, dunque, la massificazione fu particolarmente dolorosa ${ }^{31}$.

Le "classi popolari”, conclude Barbero, sebbene fossero le più indifese di fronte al processo di massificazione trovarono in esso non solamente la "possibilità di sopravvivenza fisica», ma anche le condizioni di accesso o crescita culturale. Nella cultura delle masse - conclude - queste classi trovarono «alcune delle proprie modalità di vedere il mondo, di sentirlo e di esprimerlo»32.

Nel frattempo, in contrapposizione con la letteratura predominante all'epoca, la possibilità di considerare la televisione come espressione di una nuova arte, produsse, sin dagli anni Cinquanta, alcune analisi discordanti. Da un numero ristretto di artisti e intellettuali, infatti, la televisione fu ben accolta come una promessa di rinnovamento artistico. Invece di considerarla come la tomba della lettura, la percepirono come uno spazio per la diffusione del libro e un luogo in cui si sarebbe consolidato un nuovo e stimolante mercato del lavoro, principalmente per ciò che riguardava la crescita del teleteatro, antenato delle attuali telenovelas.

A sua volta la televisione ricercava questa tipologia di intellettuali, meno refrattari alla novità tecnologica e artistica che essa rappresentava. E questi non si fecero attendere. Prodotto di un cambiamento di mentalità o della necessità di cercare nuovi ambiti lavorativi, intellettuali e artisti teatrali o cinematografici, essi entrarono a far parte dello staff delle diverse emittenti. In gran parte provenienti dalle "classi medie" a cui fa riferimento Barbero, questi professionisti portarono in televisione le loro inquietudini e speranze dinanzi alle scelte politiche e sociali che si prospettavano per i Brasiliani.

Subito dopo il golpe militare,ad esempio, artisti e scrittori - e assimilati dal mezzo - videro la televisione come uno spazio culturale che avrebbe permesso il riscatto degli ideali del nazionalismo-popolare, benché debitamente rivisti per far parte dell'industria dell'intrattenimento. La concezione di arte impegnata, che caratterizzò intensamente il dibattito politico e culturale nazionale nel passaggio tra gli anni Cinquanta e gli anni

${ }^{31}$ Ibidem, p. 223.

${ }^{2}$ Ibidem. 
Sessanta, si adattava alla cultura popolare di massa e stabiliva con essa un possibile dialogo.

In questo gruppo troviamo nomi significativi della drammaturgia impegnata, originaria dei Centros Populares de Cultura (CPCs) e simpatizzanti del Partido Comunista Brasileiro (PCB), come Gianfrancesco Guarnieri, Ferreira Gullar e Dias Gomes. Tutti loro entrarono a far parte dei quadri della TV Globo negli anni Sessanta. Dias Gomes fece la sua prima comparsa sul teleschermo nel 1953, sulla TV Tupi, dopo aver perso l’impiego a Rádio Clube do Brasil a causa della sua identificazione come comunista. Alcuni altri intellettuali legati al PCB - è il caso di Paulo Pontes e Oduvaldo Viana Filho - già erano comparsi a TV Tupi, prima emittente nazionale, fuggendo dai problemi con la censura dei decenni precedenti.

\section{Campo televisivo, campo di lotta}

La stessa incapacità di percepire la nuova dinamica in cui erano inseriti i mezzi di comunicazione di massa suscitò una certa diffidenza da parte del regime militare nei primi documenti sulle politiche pubbliche in materia di cultura. Sebbene fosse evidente una pianificazione strategica per questo campo, con la creazione del Consiglio federale della cultura, Conselho Federal de Cultura (1966), le discussioni volte ad elaborare una Política Nacional de Cultura (PCN) rivelano che gli ideologi del regime militare interpretavano aprioristicamente la cultura popolare di massa a partire da un pregiudizio basato sulle analisi tradizionali descritte in precedenza.

Per questi intellettuali, la «spontaneità della creazione popolare» stava venendo ridotta dalla «cultura di massa» così come dalla «razionalizzazione della società industriale». Stando così le cose, lo Stato, avrebbe dovuto preoccuparsi della qualità della creazione culturale, stimolando la «concorrenza qualitativa» tra gli agenti di produzione e, al contempo, combattendo il «culto della novità» caratteristico della «comunicazione di massa», che avrebbe potuto portare a una denazionalizzazione culturale 33 .

Nonostante questa sfiducia in una delle sue linee guida, la PCN evidenziava l'importanza dell'utilizzazione dei mezzi di comunicazione di massa come diffusori di valori e pratiche considerati come «espressioni dello spirito dell'uomo brasiliano» 34 . Preannunciava il fatto che i governi militari non avrebbero rinunciato alla capacità

33 BRASIL, Política Nacional de Cultura, Brasília, Departamento de Documentação e Divulgação-MEC, 1975. pp. 12-13.

34 Ibidem, p. 14. 
persuasiva mediatica - dunque produttrice di senso - di questi mezzi, specialmente la televisione, che assunse un ruolo privilegiato nel passaggio tra gli anni Sessanta e i Settanta, come dimostra la legislazione prodotta in questo periodo sulla concessione e la programmazione televisiva.

I testi che disciplinavano lo sfruttamento delle comunicazioni nel paese, anche prima dello stesso golpe civile-militare, rappresentavano una vittoria, sul piano giuridico, della concezione che veniva portata avanti dalla ESG, a partire dagli studi sulla Sicurezza e lo Sviluppo e che diedero origine alla DSN35. Nella pratica, l'esame delle telecomunicazioni in Brasile non può essere fatto senza stabilire una correlazione tra il suo sviluppo e le tesi dell' integrazione nazionale, sicurezza e sviluppo presenti nella DSN ${ }^{36}$.

Prova di questo è l'esistenza di otto articoli nella Legge di Sicurezza Nazionale, Lei de Segurança Nacional (LSN) del $1969^{37}$ che indicano l'uso dei mezzi di comunicazione di massa come un'aggravante nei casi di crimini contro la sicurezza nazionale. Oltre a ciò, la LSN concedeva alcune competenze al Conselho de Segurança Nacional per le questioni che riguardavano la politica delle telecomunicazioni, in un momento in cui propugnava la cooperazione tra questo e gli organi di informazione militare. La stessa composizione del CONTEL presupponeva la partecipazione dei rappresentanti di tre ministeri militari, oltre a più di otto membri indicati dal Capo dello Stato Maggiore delle Forze Armate.

Obiettivi Nazionali e Sicurezza Nazionale appaiono nella legislazione come concetti chiave auto-esplicativi. Categorie che, in determinate epoche, aspiravano ad aprire o

35 Fino agli anni Sessanta, le comunicazioni nel paese erano rette dalle linee direttive della Comissão Técnica do Rádio e do Ministério da Viação e Obras Públicas, transformata in Ministero dei Trasporti, nel 1967. Nel maggio del 1961 fu creato il Conselho Nacional de Telecomunicações (CONTEL) che, nell'anno successivo, iniziò a disciplinare questi servizi attraverso il Codice Brasiliano delle Telecomunicazioni (CBT). Il CBT stabilì la competenza dell'Unione federale nello sfruttamento diretto, o tramite concessione, del servizio di radiodiffusione sonora (regionale o nazionale) e di quello televisivo. Il rilascio della concessione o dell'autorizzazione divenne prerogativa del presidente della Repubblica, previa la consultazion del CONTEL. È importante sottolineare che tutta questa legislazione sorse nel bel mezzo della crisi del governo di João Goulart, e, in un certo modo, dimostrò la debolezza delle proposte presentate dall'Esecutivo. Il Codice rifletteva lo sforzo comune dei due gruppi di interesse: i militari, che già si erano dedicati allo sviluppo di questo settore ritenendolo uno strumento di progresso e gli imprenditori della radiodiffusione che avevano gettato le basi dell'Associaciazione brasiliana della radiotelevisione, Associação Brasileira de Rádio e Televisão (ABERT) nello stesso anno. Questi ultimi coltivavano la speranza che la legislazione potesse limitare l'interferenza del Potere esecutivo sui loro affari, consolidando in via definitiva la predominaznza dell'iniziativa privata nel modello che si stava impiantando.

${ }^{36}$ PRIOLLI, Gabriel, A tela pequena no Brasil Grande, in LIMA, Fernando Barbosa, PRIOLLI, Gabriel, MACHADO, Arlindo. Televisão \& Vídeo, Rio de Janeiro, Jorge Zahar Ed., 1989, pp. 1952.

${ }^{37}$ Stabilita con il Decreto-legge n. 898, del settembre 1969. 
chiudere qualche porta, utilizzate come espressioni che costituiscano un valore in se stesso e non come simboli, che invece esprimono un significato. Secondo Sérgio Caparelli, in termini linguistici, si potrebbe dire che il significante inghiotte il significato e desidera fare storia, ma fuori da questa ${ }^{3}$.

L'enunciazione di una politica ufficiale delle comunicazioni da parte del regime militare tanto nella sua istanza culturale quanto nell'offerta di tecnologia sofisticata all'iniziativa privata ${ }^{39}$, venne a comporre un progetto egemonico che accompagnando e rendendo possibile la crescita di altri settori dell'economia nazionale, rese concrete la legittimazione e la definitiva istituzionalizzazione del regime.

Anche se già il programma di governo del generale Costa e Silva (1967-1969) presentava le Comunicazioni come un settore strategico ${ }^{40}$, fu solo nel mandato di Garrastazu Médici (1969-1974), durante il cosiddetto "Miracolo Economico", che si percepì come l'utilizzo della comunicazione di massa servisse ad una amministrazione che si manteneva sostanzialmente in base all'adozione di strumenti prodotti dallo stato d'eccezione. Il documento "Obiettivi e basi per l'azione del governo" sottolinea che

[...] Lo sviluppo integrato, [...], a livelli tanto elevati, sarà possibile solamente attraverso l'instaurazione di processi politici riduttivi della violenza del conflitto sociale proprio «della società divisa in classi, bloccando i canali di espressione legittimi, soprattutto delle classi subalterne $[\ldots]^{41}$ [corsivo dell'autrice].

L'efficacia della cultura popolare di massa per l'accettazione sociale dell'instaurazione [di questi] processi politici riduttivi porterà i governi militari, particolarmente quelli dei generali Médici e Geisel, a intervenire in modo più diretto sul contenuto dei programmi televisivi nazionali. La televisione doveva servire per l'affermazione di nuove abitudini, valori e comportamenti compatibili con il progetto

${ }^{8} 8$ CAPARELLI, Sérgio, Televisão e capitalismo no Brasil, Porto Alegre, L\&M Editores, 1982.

39 Perché la televisione si trasformasse in uno dei più importanti veicoli di diffusione della dottrina del regime militare le mancava la portata nazionale. All'esigenza si venne incontro grazie all'Empresa Brasileira de Telecomunicações (Embratel) nel 1965. Una delle sue prime funzioni fu quella di impiantare, espandere e mettere in opera le strutture televisive basilari, oltre alle stazioni terrestri necessarie per diffondere le comunicazioni nazionali via satellite. Questo compito faceva parte del progetto di integrazione nazionale dei governi militari e ben presto fu fatto il primo passo con l'inaugurazione della Rete di base per microonde (1969), garantendo una comunicazione efficace per la telefonia, oltre alle trasmissioni della televisione, della radio e di dati tra le diverse aree del paese. Questa tecnologia garantì l'iniziativa privata nel campo televisivo e l'infrastruttura necessaria per il suo sviluppo.

$4^{\circ}$ MINISTÉRIO DO PLANEJAMENTO E COORDENAÇÃO GERAL DO BRASIL, Diretrizes do Governo - Programa Estratégico de Desenvolvimento, Brasília, Ministério do Planejamento e Coordenação Geral, 1967, p. 16.

${ }^{41}$ PRESIDÊNCIA DA REPÚBLICA, Metas e Bases para a Ação de Governo - Brasil, Brasília, Presidência da República, 1970. 
nazionale di sviluppo congiunto alla sicurezza. La sua programmazione doveva costituirsi in «un nucleo irriducibile di cultura autonoma che potesse imprimere un aspetto proprio al tenore di vita brasiliano»42.

Più che il riconoscimento immediato per gli atti del governo, i gruppi di potere dell'epoca cercavano nella programmazione televisiva un aiuto efficace per la produzione di significati desiderabili per il Brasile e per il brasiliano. Questo implica dire che gli ideologi di questi gruppi iniziarono a valorizzare i fenomeni culturali, ivi compresi quelli di massa, per la loro capacità - idealizzata dalla DSN - di unificare il mercato materiale e simbolico e di rendere concreta l'istituzionalizzazione del regime.

È importante affermare che questo non era un progetto esclusivo dei settori militari. Guardare la televisione non significava solamente avere accesso ai messaggi espliciti della sua programmazione. Tutta la capacità persuasiva del veicolo permette di avere accesso (benché, alcune volte, solo a livello di desiderio) ai beni materiali e simbolici veicolati dalle pubblicità o dal merchandising. Durante la dittatura militare, nonostante l'incremento della disuguaglianza economica, la televisione fu in grado di formare il cittadino consumatore, soprattutto fra i settori in ascesa della classe media urbana, rispondendo così positivamente al progetto di sviluppo che univa lo Stato e il grande capitale privato nazionale e internazionale.

Tuttavia la capacità di unificare il mercato materiale e simbolico dimostrata dalla televisione brasiliana, in particolare negli anni Settanta, non può essere analizzata come una strada a senso unico. In quanto parte della lotta per l'egemonia che si stabilì all'interno e all'esterno dei gruppi di potere, questa unificazione si costituì quotidianamente a partire dal conflitto fra settori differenti che non agivano solamente sulla base del loro posizionamento "di classe", ma anche in ragione di valutazioni soggettive dei limiti e possibilità che si presentavano in un continuo movimento dialettico dove erano presenti forze di coazione, di consenso e di compromesso 43 .

A titolo d'esempio, indichiamo rapidamente il posizionamento dell'imprenditoria del campo televisivo ${ }^{44}$, in quanto partecipante al gruppo di potere durante la dittatura

42 MINISTÉRIO DA EDUCAÇÃO E CULTURA DO BRASIL, Política Nacional de Cultura, Brasília, Departamento de Documentação e Divulgação-MEC, 1975. p. 28.

43 Cfr. GRAMSCI, Antonio, Cadernos do Cárcere, vol. 3, Rio de Janeiro, Civilização Brasileira, 2000.

44 Si considera il concetto di campo per come lo ha teorizzato da Pierre Bourdieu: «uno spazio sociale strutturato, un campo di forze - dove ci sono dominanti e dominati, dove vi sono relazioni costanti, permanenti, di disuguaglianza, che si esercitano all'interno di questo spazio che è anche un campo di lotta per trasformare o conservare questo campo di forze». BOURDIEU, Pierre, Sobre a televisão, Rio de Janeiro, Jorge Zahar, 1997, p. 57. Così il campo televisivo viene interpretato come uno spazio di lotta dove si intrecciano cultura e politica nella produzione di significati. 
militare. Come rappresentanti del grande capitale, anche se non occupavano cariche pubbliche nel governo o nello Stato, questi attori sociali detenevano posizioni di potere sfruttando l'importanza che il campo finì per acquisire - in quanto elemento di legittimazione del regime - per far prevalere i propri interessi45.

Tuttavia non si deve considerare l'espressione gruppo di potere come sinonimo dell'esistenza di una corrispondenza illimitata tra gli interessi degli imprenditori e il regime militare, perché questa sarebbe una posizione eccessivamente semplicistica. Allo stesso modo non si prende in considerazione il fatto che gli imprenditori avevano obiettivi non sempre coincidenti. Questo non gli impediva, comunque, di agire politicamente insieme in difesa dei propri interessi economici o di determinati valori sociali. Gruppo di potere, pertanto, è qui utilizzato come categoria analitica per esprimere un insieme di relazioni che si costituiscono giorno per giorno, in base alle difficoltà e alle condizioni che storicamente si presentano.

Prenderemo come esempio la posizione illustrata qui sotto dall'imprenditore Roberto Marinho, concessionario della TV Globo, una delle emittenti più importanti e maggiormente seguita a quell'epoca:

[...] e nella misura in cui l'iniziativa privata in Brasile, nel campo della comunicazione trovò la competenza anzitutto per raggiungere il mercato, successivamente per conquistarne la fiducia attraverso la qualità dei prodotti offerti, ora può iniziare a offrire prodotti maggiormente elaborati in vista del miglioramento culturale. Nella misura - io direi - in cui l'iniziativa privata trovò la competenza necessaria per domare un mezzo nuovo e complesso come la televisione, essa sta realizzando un lavoro di altissimo senso pubblico, libera dalle pressioni a cui lo Stato, inevitabilmente, è sottoposto ${ }^{46}$.

E ancora:

Siamo noi, [...] comunicatori, una sorta di agenti delegati della stabilità pubblica. Dalla nostra attività dipendono le modalità attraverso cui tranquillizzare la popolazione [...]. [Gli] uomini della comunicazione, quelli che li garantiscono, nel loro spirito libertario, contro qualsiasi attacco statalista o totalitario, quelli che

\footnotetext{
45 Cfr. REZENDE, Maria José de, A ditadura militar no Brasil: repressão e pretensão de legitimidade: 1964-1984, Londrina, Ed. UEL, 2001, pp. 8-9.

${ }^{46}$ MARINHO, Roberto, $O$ sentido público da atividade privada na comunicação, intervento presentato alla Federação Nacional das Empresas de Seguros Privados e de Capitalização, il 17 maggio 1978. Cit. in TAVARES, Zulmira Ribeiro, A alma do negócio: vozes da/sobre a televisão, testo ciclostilato archiviato nel Museu da Imagem e do Som, Rio de Janeiro.
} 
garantiscono un servizio che rispetta profondamente il sentimento, il pensiero e l'emozione del pubblico47.

Dunque è vero che la televisione brasiliana dell'epoca, per la capacità di mediazione dimostrata, si rivelò essere il primo luogo in cui modellare ideologicamente la società. Tuttavia le strategie di negoziazione simbolica che stabilì con il suo pubblico non lasciarono che si trasformasse in un semplice apparato dello Stato, benché questo fosse autoritario. Così solo l'esame dell'interazione tra significato e potere nelle specifiche circostanze storico-sociali permetterà di capire se determinati fenomeni simbolici innescati dalla televisione siano serviti o meno per stabilire o mantenere rapporti di dominio ${ }^{48}$.

Analizzare la specificità dei valori e degli interessi delle classi, dei gruppi e dei segmenti sociali come relazioni che si sono consolidate storicamente e non come un dato di fatto a priori significa prendere in considerazione l'esistenza di letture differenti, fatte a partire da comunità di significati distinte; significa percepire il consolidamento di queste comunità, anche ideologiche, come un eterna operazione di ricostruzione.

\footnotetext{
${ }^{47}$ Ibidem.

${ }^{48}$ THOMPSON, John B., Ideologia e Cultura Moderna - teoria social crítica na era dos meios de comunicação de massa, Petrópolis, Rio de Janeiro, Vozes, 1995.
} 


\section{* L'autore}

Sonia Wanderley si è addottorata in Storia sociale presso l'Universidade Federal Fluminense (UFF). Attualmente è professoressa dell'Universidade do Estado do Rio de Janeiro (UERJ), dove lavora all'interno dell'Instituto de Aplicação (CAp/UERJ), oltre che nel corso di Laurea magistrale in Storia sociale della Facoltà di Scienze della formazione (FFP/UERJ). Fa parte anche del corpo docente del corso di Laurea magistrale di insegnamento della Storia (ProfHist UERJ) e dei gruppi di ricerca Oficinas de História e Laboratório de Estudo das Diferenças e Desigualdades (LEDDES).

URL: < http://www.studistorici.com/progett/autori/\#Wanderley >

\section{Per citare questo articolo:}

WANDERLEY, Sonia, «Dittatura militare e televisione in Brasile: una rilettura critica (1964-1979)», Diacronie. Studi di Storia Contemporanea : Le dittature militari: fisionomia ed eredità politica, 29/12/2015,

URL:<http://www.studistorici.com/2015/12/29/wanderley_numero_24/ >

Diacronie Studi di Storia Contemporanea $\vartheta$ www.diacronie.it

Risorsa digitale indipendente a carattere storiografico. Uscita trimestrale.

redazione.diacronie@hotmail.it

Comitato di redazione: Jacopo Bassi - Luca Bufarale - Elisa Grandi - Antonio César Moreno Cantano - Deborah Paci - Fausto Pietrancosta - Alessandro Salvador - Matteo Tomasoni - Luca Zuccolo

Diritti: gli articoli di Diacronie. Studi di Storia Contemporanea sono pubblicati sotto licenza Creative Commons 3.0. Possono essere riprodotti e modificati a patto di indicare eventuali modifiche dei contenuti, di riconoscere la paternità dell'opera e di condividerla allo stesso modo. La citazione di estratti è comunque sempre autorizzata, nei limiti previsti dalla legge. 